\title{
Posh splicing
}

\section{By Michael J. Haas, Senior Writer}

North Carolina researchers have designed splicing factors that could help treat cancer and other diseases by selectively promoting or inhibiting the expression of specific isoforms of their target genes. ${ }^{1}$ The technology offers potential advantages over existing therapies that target gene splicing, including longer-lasting effects and the ability to hit any gene.

A single gene may express different isoforms of a protein depending on how the gene's transcripts are assembled (spliced) into a single mRNA molecule. Moreover, splicing isoforms can play differing-even opposite-roles in cellular function. For example, the $B c l-X L$ gene codes for both a splicing isoform that is involved in normal mitochondrial apoptosis and an antiapoptotic splicing isoform that is upregulated in many cancers.

"This is a very elegant, imaginative and novel approach to build an artificial splicing factor from scratch."

-Ryszard Kole, AVI BioPharma Inc.
The team was led by Zefeng Wang, assistant professor of pharmacology at UNC Chapel Hill, and included other UNC Chapel Hill researchers and Traci Hall, principal investigator at NIEHS and leader of the 2006 study reported in PNAS.

"This is a very elegant, imaginative and novel approach to build an artificial splicing factor from scratch," said Ryszard Kole, SVP of discovery research at AVI BioPharma Inc. "They essentially create novel components of the cellular splicing machinery. By doing so, they mimic the effects that were previously achieved by targeting splice sites with antisense oligonucleotides."

AVI is developing antisense agents, called phosphorodiamidate morpholino oligomers (PMOs), to treat Duchenne muscular dystrophy (DMD), Ebola virus and Marburg virus infections, and restenosis.

"There is a gap in the tools available to study the transcriptosome," said Vin Kotraiah, associate director of collaborative therapeutics at ExonHit Therapeutics Inc., the U.S. subsidiary of ExonHit Therapeutics S.A. "We can identify alternative splicing events but don't have good tools to study the consequences of alternative splicing events in a controlled setting."

ExonHit utilizes its SpliceArray technology to identify splicing isoforms involved in disease. The company is developing small molecule therapeutics targeting those isoforms to treat Alzheimer's disease (AD), cancer and epilepsy, and has a deal with Allergan Inc. to develop therapeutics against targets identified by monitoring alterations in splicing isoforms to treat pain, neurodegeneration and ophthalmic indications.

\section{Genetic advantages}

Kole said the ESFs have both advantages and drawbacks compared with antisense molecules. On the plus side, he said, ESFs "could be delivered with lentiviral vectors and incorporated into the genome, and so could have longer-lasting or even permanent effects compared with antisense oligonucleotides."

But he added that ESF technology would introduce a foreign protein into the body, creating the potential for an adverse immune response.

"The next logical step is to test the approach in vivo in appropriate animal models," Kole told SciBX.

Kotraiah said ESFs might have two additional advantages over antisense agents or small molecules that target gene splicing.

"Antisense oligonucleotides are primarily used to block certain splicing sites," he said. "ESFs are more versatile because they can block or promote splicing" on the target gene.

Compared with small molecules, ESFs might have a broader range of gene targets, noted Kotraiah. "It might be difficult to target every alternative splicing event with a small molecule that interacts with a protein upstream in the splicing pathway," he said. "If this technology works as the authors think it does, ESFs could directly target any splicing event." 


\section{Splice mining}

Nevertheless, Kotraiah said ExonHit is more interested in the immediate potential of ESFs as tools to study splicing isoforms as drug targets rather than using ESFs as therapeutics.

Thus, he thought ESF technology and ExonHit's SpliceArray platform could complement each other. "SpliceArray can identify alternative splicing events that occur in diseased versus healthy cells," he said. "Then you could follow on with ESF technology to induce the expression of alternatively spliced isoforms in models and study their functions. Conversely, off-target effects of ESFs on alternative splicing and gene expression could be monitored on a transcriptome-wide scale using SpliceArrays."

He added: "We would definitely be interested in keeping an eye on this technology and how it develops."

On the heels of the results described in Nature Methods, Wang said his team has designed ESFs that target undisclosed apoptosis-related genes involved in cancer and is studying an ESF that targets an angiogenic gene in animal models of cancer.

The in vivo studies are being conducted by Yang Wang, a postdoctoral associate in Zefeng Wang's group who invented the ESF technol- ogy and was lead author on the Nature Methods paper.

UNC Chapel Hill and NIEHS have filed patent applications covering the use of ESF technology as a drug platform and as a tool to study gene splicing, and the technology is available for licensing. "If other people are interested, we would certainly welcome collaborations to develop applications" for ESFs, Zefeng Wang said.

Haas, M.J. SciBX 2(40); doi:10.1038/scibx.2009.1493

Published online Oct. 15, 2009

\section{REFERENCES}

1. Wang, Y. et al. Nat. Methods; published online Oct. 4, 2009; doi:10.1038/nmeth.1379

Contact: Zefeng Wang, The University of North Carolina at Chapel Hill, Chapel Hill, N.C. e-mail: zefeng@med.unc.edu

2. Cheong, C. \& Hall, T. Proc. Natl. Acad. Sci. USA 103, 13635-13639 (2006)

\section{COMPANIES AND INSTITUTIONS MENTIONED}

Allergan Inc. (NYSE:AGN), Irvine, Calif.

AVI BioPharma Inc. (NASDAQ:AVII), Bothell, Wash.

ExonHit Therapeutics S.A. (Euronext:ALEHT), Paris, France

National Institute of Environmental Health Sciences, Research

Triangle Park, N.C.

The University of North Carolina at Chapel Hill, Chapel Hill, N.C. 